\section{Avaliação do impacto do Programa Nacional do Leite em Alagoas, através de métodos isotópicos: aspectos metodológicos e resultados preliminares}

\section{Isotope methods for evaluating the impact of the National Nutritional Program in Alagoas: methodological aspects and preliminary results}

\author{
Denise Petrucci Gigante \\ Programa de Pós-graduação em Epidemiologia \\ Universidade Federal de Pelotas \\ Departamento de Nutrição - UFPel \\ Endereço para correspondência/Correspondence to: \\ Campus Universitário, s/nº - Caixa Postal 354 \\ 96010-900 - Pelotas, RS - Brasil \\ denise@epidemio-ufpel.org.br
}

Iná S. Santos

Programa de Pós-graduação em Epidemiologia - UFPel

Departamento de Medicina Social - UFPel

\section{Denise Costa Coitinho}

Área Técnica de Alimentação e Nutrição

Ministério da Saúde

Neiva C.J.Valle

Programa de Pós-graduação em Epidemiologia - UFPel

Departamento de Estatística, Matemática e Computação - UFPel

\section{Hinke Haisma}

Programa de Pós-graduação em Epidemiologia - UFPel

\section{Gicele C. M. Valente}

Programa de Pós-graduação em Epidemiologia - UFPel

\section{Auxílio Financeiro}

Ministério da Saúde - Fundação Nacional de Saúde - Processo nº 964/00

\section{Resumo}

Objetivos: Descrever aspectos metodológicos e resultados preliminares de um estudo de intervenção planejado para medir o impacto de um programa de suplementação alimentar sobre a promoção do crescimento infantil de crianças desnutridas. Métodos: Estudo longitudinal, controlado, desenvolvido no estado de Alagoas, com grupos comparados antes e depois da intervenção. Vinte municípios foram selecionados através de um indicador de cobertura do programa de suplementação alimentar. Essa cobertura foi estimada pela diferença entre a proporção de crianças desnutridas e a proporção de crianças atendidas pelo programa. Os dez municípios com indicador de alta cobertura foram selecionados para o grupo intervenção e aqueles de menor cobertura para o grupo controle. Em cada município do grupo intervenção foram selecionadas 15 crianças ingressando no Programa. Em cada município do grupo controle foram incluídas 15 crianças elegíveis, mas que não conseguiram vaga no Programa. Resultados: Foram aplicados 111 e 102 questionários em crianças dos municípios do grupo intervenção e controle, respectivamente. A comparação em relação às características de base mostra que a distribuição das variáveis demográficas e nutricionais foi semelhante entre as crianças dos grupos intervenção e controle. Por outro lado, as crianças do grupo intervenção são provenientes de famílias com menor renda familiar e seus pais apresentaram menor escolaridade quando comparadas às do grupo controle. Conclusões: Os resultados preliminares mostram que os grupos são comparáveis em relação às variáveis demográficas e nutricionais e que as diferenças observadas quanto ao nível socioeconômico deverão ser consideradas na análise ajustada, na segunda fase do estudo.

Palavras chave: Estudos de intervenção. Avaliação de programas. Suplementação alimentar. Criança. 
Abstract

Objective: To describe methodological aspects and preliminary results of an intervention designed to measure the impact on child growth of nutritional programs delivered to underweight children in a Northeastern state of Brazil. Methods: This is a natural longitudinal controlled experiment or quasi experiment, in which intervention and control groups were compared at baseline and endpoint. Twenty municipalities of the state of Alagoas were selected through a coverage indicator of the nutritional program at the municipal level. Coverage was estimated by the difference between the proportion of underweight children and the proportion of children actually reached by the nutritional program. The ten municipalities with higher coverage indicators were selected for the intervention group, those ten with lower coverage were selected for the control group. Fifteen children that entered the program in each intervention municipality and fifteen eligible children not included in the nutritional program were included in the study. Results: A structured questionnaire and nutritional assessment were applied on 111 children of the intervention group and 102 children of the control group. Baseline characteristics are presented. The demographic variables for mothers and children were similar in the intervention and control group. Children from the intervention group had lower family income and their parents had a lower level of schooling. There was no difference in the nutritional characteristics of the intervention and control group. Conclusion: The preliminary results showed that the groups are comparable in terms of demographic and nutritional variables. The difference in the socioeconomic level between intervention and control group will be considered in the adjusted analyses in the second phase of the study.

Keywords: Intervention studies. Program evaluation. Supplementary feeding. Child.

\section{Introdução}

Nas duas últimas décadas houve uma diminuição nas prevalências de desnutrição em todo país. Entretanto essa diminuição não ocorreu em todas as regiões da mesma forma (PNSN, 1989) ${ }^{1}$. Nas regiões menos desenvolvidas do país, e nessas, nas áreas de menor nível socioeconômico, ainda são encontradas elevadas prevalências de desnutrição, especialmente entre as crianças até 2 anos de idade.

Os resultados da Pesquisa Nacional Sobre Demografia e Saúde (PNDS, 1996) ${ }^{2}$ mostram que $17,9 \%$ das crianças nordestinas menores de 5 anos de idade apresentam déficit de estatura para a idade e 8,3\% apresentam déficit de peso para a idade, considerando como ponto de corte 2 desvios padrão da referência do National Center of Health Statistics ${ }^{3}$.

O Programa Nacional de Incentivo ao Combate às Carências Nutricionais (ICCN) tem como objetivo o atendimento de crianças em risco nutricional na faixa etária entre 6 e 23 meses mediante o fornecimento de leite e óleo de soja. Embora se tratando de uma iniciativa nacional, cuja responsabilidade é da Secretaria de Políticas de Saúde, através da Área Técnica de Alimentação e Nutrição, sua implementação é descentralizada e os municípios têm autonomia de decidir sua participação ou não no Programa.

De acordo com as diretrizes do Programa, toda criança com idade entre 6 e 23 meses com peso abaixo da linha inferior do Cartão da Criança (Percentil 10) é considerada em risco nutricional. Essas crianças, ao serem selecionadas para inclusão no Programa, deverão receber um litro de leite integral (ou $120 \mathrm{~g}$ de leite em pó integral) por dia e uma lata de óleo de soja por mês. O leite e o óleo deverão ser adquiridos pelo município de acordo com especificação do Ministério da Saúde. A suplementação alimentar deve ser fornecida por pelo menos 12 meses, as crianças devem ser pesadas mensalmente e os dados devem ser registrados no Sistema de Vigilância Alimentar e Nutricional (SISVAN) do município. Para 
cada município do país foi estimado o número de crianças entre 6 e 23 meses em risco nutricional a partir de um modelo estatístico de predição do estado nutricional elaborado pelo Núcleo de Pesquisas em Nutrição e Saúde da Universidade de São Paulo (NUPENS/USP).

O impacto do Programa sobre o crescimento das crianças e o grau de adesão a essa intervenção nutricional, no entanto, nunca foram avaliados no Brasil. Considerando que o principal objetivo de uma avaliação é influir sobre a tomada de decisões ${ }^{4}$, tornamse extremamente necessárias informações que indiquem a efetividade do Programa e as possíveis razões para falhas no alcance de seus objetivos.

Este artigo se propõe a descrever o processo metodológico para a realização de uma avaliação desse tipo em um estado da região nordeste do país, área em que a desnutrição e a pobreza são mais prevalentes. Esse estudo está sendo desenvolvido no estado de Alagoas e pretende estimar a efetividade do Programa Nacional de Incentivo ao Combate às Carências Nutricionais sobre a promoção do crescimento infantil e medir a adesão materna ao uso do alimento suplementar conforme a recomendação proposta. Dessa forma, serão comparadas as diferenças nos índices nutricionais durante o período de seis meses, além da comparação da composição corporal através de avaliação da água corporal total pelo método de diluição do deutério. Ainda nesse artigo serão apresentados resultados iniciais da comparação dos indicadores do estudo de base.

\section{Metodologia}

O estudo apresenta um delineamento longitudinal, controlado, com as áreas incluídas no programa de suplementação alimentar e controle sendo comparadas antes e depois da intervenção. Os municípios incluídos foram selecionados com base nos indicadores nutricionais obtidos a partir de modelos de regressão logística multivariada que utilizaram dados demográficos, socioeconômicos e ambientais. A amostra foi constituída pelos dez municípios com mais alta cobertura do Programa Nacional de Incentivo ao Combate às Carências Nutricionais e outros dez, com tamanho e estrutura semelhantes onde a suplementação alimentar apresentava as mais baixas coberturas. Os vinte municípios foram selecionados de acordo com a diferença entre as prevalências estimadas de desnutrição e o número de crianças atendidas pelo Programa Nacional de Incentivo ao Combate às Carências Nutricionais.

Para o cálculo do tamanho da amostra, o nível de significância foi estabelecido em $5 \%$ (unicaudal), o poder em $80 \%$ e, a unidade de estudo foi definida como cada um dos municípios. No cálculo considerou-se 0,17 kg como desvio padrão das diferenças de ganho de peso no período de seis meses entre as crianças residentes na região de cobertura dos serviços de saúde de acordo com estudo prévio realizado em uma coorte de crianças nascidas em Pelotas, em $1993^{5}$. Para que se possa detectar uma diferença de 150 g em ganho de peso entre o grupo intervenção e controle, foi necessária a inclusão de dez municípios em cada grupo.

Em cada município foram identificadas as mães e suas crianças de 6 a 18 meses de idade que eram atendidas nos postos de saúde municipais. Foram selecionadas para o estudo as primeiras 15 crianças que ingressaram no Programa Nacional de Incentivo ao Combate às Carências Nutricionais em cada área de intervenção. Nos municípios controle, 15 crianças abaixo do percentil 10 (índice peso/idade) identificadas nos serviços, monitorizadas por trabalhadores de saúde e que não estavam participando do Programa, foram escolhidas como controles.

Ao serem incluídas no estudo, e seis meses mais tarde, as crianças de ambos os grupos, intervenção e controle, foram pesadas sem roupas em de balança portátil com precisão de 100g (CMS PBW-25; London, UK) e o seu comprimento foi aferido por antropômetros (modelo AHRTAG baby length measures; London, UK). As técnicas de tomadas de medidas de peso e comprimento 
foram usadas conforme recomendação de Lohman e colaboradores ${ }^{6}$ e houve treinamento e padronização entre os trabalhadores de campo.

Também nos dois acompanhamentos, as crianças de ambos os grupos (intervenção e controle) receberam uma dose de $1 \mathrm{~g}$ de deutério. As doses foram preparadas e distribuídas em frascos individuais a serem ingeridos por cada criança. Também foram preparados três frascos, com o número de identificação de cada criança, para coleta e armazenamento de amostras de saliva. No domicílio, após o consentimento informado da mãe, uma amostra de $2 \mathrm{ml}$ da saliva foi coletada antes da ingestão da dose padrão de deutério, e outras duas amostras foram coletadas 3 e 4 horas após a ingestão. As amostras foram armazenadas a uma temperatura em torno de $-3^{\circ} \mathrm{C}$ e serão encaminhadas para análise, com vistas à determinação da composição corporal ${ }^{7}$. Esse método consiste na determinação da quantidade total de água corporal a partir de uma dose conhecida de deutério. Admitindo-se um percentual fixo de água corporal, e que a gordura não contém água, é possível estimar a massa livre de gordura e, por diferença da massa corporal total, a quantidade de gordura corporal.

Questionários estruturados foram elaborados para as duas fases do estudo. No acompanhamento de base foram incluídas informações socioeconômicas e demográficas das crianças e de suas famílias. Práticas da alimentação das crianças, investigadas através de recordatório de 24 horas e freqüência de consumo semanal, foram incluídas nos dois questionários (estudo de base e acompanhamento seis meses depois). O questionário da segunda fase do estudo também incluiu informações quanto à disponibilidade e utilização de produtos fornecidos por programas de suplementação alimentar.

Esse estudo foi aprovado pela Comissão de Ética da Faculdade de Medicina da Universidade Federal de Pelotas e pela Secretaria de Saúde do Estado de Alagoas e todos os procedimentos com as crianças só foram realizados após o consentimento in- formado da mãe ou responsável pela criança examinada.

As medidas de desfecho incluem a evolução do peso, comprimento e composição corporal de cada criança e a adesão da mãe ao uso do suplemento conforme a recomendação. A adesão materna será obtida através do relato da mesma e da observação e registro da quantidade de leite e óleo do Programa, disponíveis no município, por ocasião do segundo acompanhamento.

Os grupos de intervenção e controle foram comparados em termos dos indicadores de base, incluindo características demográficas, socioeconômicas e nutricionais. Quaisquer diferenças significativas na entrada do estudo terão seu efeito ajustado na análise. Os dois grupos serão comparados em termos de ganho de peso e comprimento. Todas as análises serão realizadas através do uso de um modelo de análise multiníveis. O nível mais alto será constituído pelos municípios, o segundo e terceiro serão constituídos, respectivamente, pelos Postos e pelos agentes de saúde e o quarto nível será constituído pelas mães e suas crianças. Essa abordagem permite que a interdependência existente entre as crianças que vivem no mesmo município, que consultam no mesmo Posto e são atendidas pelo mesmo agente de saúde seja levada em conta. Os dados foram digitados duas vezes no programa Epi-info 6.0, permitindo que se fizesse a comparação e correção de possíveis erros de digitação. Ainda nesse programa foram calculados os escores-Z para os índices peso/idade, comprimento/idade e peso/comprimento. A comparação das características das crianças entre os dois grupos foi realizada no programa SPSS 8.0 e a análise multiníveis será realizada através do programa MLWin 1.10 (Multilevel Models Project, Institute of Education, London, UK).

\section{Resultados}

Na primeira fase do estudo foram incluídas 111 crianças inscritas dos dez municípios com alta cobertura do Programa ICCN (grupo intervenção) e 102 crianças do grupo 
controle. A tabela 1 mostra a distribuição da população estudada em relação às características demográficas e socioeconômicas das crianças incluídas em cada grupo (intervenção e controle). Observa-se que não houve diferença quanto à distribuição por sexo e idade das crianças dos grupos intervenção e controle, mostrando equilibrada distribuição entre meninos e meninas e nos grupos de 6 a 11 meses e de 12 a 18 meses. O percentual de mães adolescentes $(<20$ anos) foi quase duas vezes maior no grupo controle do que no grupo intervenção; entretanto, essa diferença não foi estatisticamente significativa. Um terço das mães do grupo intervenção tinha quatro ou mais filhos, mas não houve diferença no número de irmãos da criança incluída em cada um dos grupos. Em relação às variáveis socioeconômicas estudadas observa-se que há diferença estatisticamente significativa quanto à renda familiar e à escolaridade paterna, mostrando

Tabela 1 - Características demográficas e socioeconômicas das crianças incluídas no grupo controle e intervenção. Alagoas, 2001.

Table 1 - Demographic and socioeconomic characteristics of the children included in the control and intervention groups. Alagoas, 2001.

\begin{tabular}{|c|c|c|c|}
\hline Variável & Intervenção (\%) & Controle (\%) & $p^{*}$ \\
\hline Sexo & & & 1,00 \\
\hline Masculino & $50(45,0)$ & $46(45,1)$ & \\
\hline Feminino & $61(55,0)$ & $56(54,9)$ & \\
\hline Idade & & & 0,7 \\
\hline$<12$ meses & $53(47,7)$ & $52(51,0)$ & \\
\hline 12 meses ou mais & $58(52,3)$ & $50(49,0)$ & \\
\hline Idade materna & & & 0,17 \\
\hline$<20$ anos & $17(15,3)$ & $28(27,5)$ & \\
\hline 20 a 24 anos & $35(31,5)$ & $28(27,5)$ & \\
\hline 25 a 29 anos & $16(14,4)$ & $18(17,6)$ & \\
\hline 30 a 34 anos & $20(18,0)$ & $13(12,7)$ & \\
\hline 35 anos ou mais & $23(20,7)$ & $15(14,7)$ & \\
\hline Número de irmãos & & & 0,4 \\
\hline Nenhum & $24(21,6)$ & $29(28,4)$ & \\
\hline 1 & $27(24,3)$ & $26(25,5)$ & \\
\hline 2 & $23(20,7)$ & $23(22,5)$ & \\
\hline 3 ou mais & $37(33,3)$ & $24(23,5)$ & \\
\hline Renda familliar & & & 0,03 \\
\hline Até $0,5 \mathrm{SM}$ & $36(33,3)$ & $23(22,5)$ & \\
\hline 0,5 a $0,9 \mathrm{SM}$ & $28(25,9)$ & $27(26,5)$ & \\
\hline 1 a $1,4 \mathrm{SM}$ & $30(27,8)$ & $23(22,5)$ & \\
\hline $1,5 \mathrm{SM}$ ou mais & $14(13,0)$ & $29(28,4)$ & \\
\hline Escolaridade materna & & & 0,18 \\
\hline Analfabeta & $54(49,5)$ & $38(38,0)$ & \\
\hline 1 a 3 anos & $28(25,7)$ & $27(27,0)$ & \\
\hline 4 anos ou mais & $27(24,8)$ & $35(35,0)$ & \\
\hline Escolaridade paterna & & & 0,02 \\
\hline Analfabeto & $53(58,9)$ & $32(38,6)$ & \\
\hline 1 a 3 anos & $20(22,2)$ & $23(27,7)$ & \\
\hline 4 anos ou mais & $17(18,9)$ & $28(33,7)$ & \\
\hline
\end{tabular}

* Qui-quadrado de Pearson/ Person's chi-square test 
que no grupo intervenção há um maior número de crianças pertencentes a famílias com menor renda familiar mensal e que há um maior percentual de pais analfabetos nesse grupo em relação ao grupo controle. Ainda nessa tabela pode ser observado que não havia informação sobre a escolaridade paterna em $18,8 \%$ da população estudada, entretanto essa falta de informação foi semelhante entre os grupos, em 21 crianças do grupo intervenção $(18,9 \%)$ e em 19 crianças do grupo controle (18,6\%).

A comparação das crianças dos dois grupos em relação às características nutricionais é mostrada na Tabela 2. Observa-se que houve uma diferença estatisticamente significativa quanto ao peso de nascimento das crianças, mostrando maior prevalência de baixo peso ao nascer entre as crianças dos municípios controle. Por outro lado, ao analisar a média do peso de nascimento observa-se que essa é maior entre as crianças do grupo controle, sem que haja uma diferença estatisticamente significativa em relação à média das crianças do grupo intervenção. Ainda quando são comparados os indicadores nutricionais no momento em que essas crianças foram incluídas no estudo, não há diferença para qualquer um dos índices. Há uma maior proporção de crianças consumindo leite materno atualmente no grupo intervenção, mas essa diferença não é significativa.

\section{Discussão}

A impossibilidade de cobertura do Programa Nacional de Incentivo ao Combate às Carências Nutricionais a todas as crianças com déficit de peso/idade em municípios do estado de Alagoas permitiu que se realizasse esse estudo de intervenção denomi-

Tabela 2 - Características nutricionais das crianças incluídas no grupo controle e intervenção. Alagoas, 2001.

Table 2 - Nutritional characteristics of the children included in the control and intervention groups. Alagoas, 2001.

\begin{tabular}{|c|c|c|c|}
\hline Variável & Intervenção (\%) & Controle (\%) & $\mathrm{p}^{*}$ \\
\hline Peso ao nascer & & & 0,007 \\
\hline Menor que $2500 \mathrm{~g}$ & $12(13,5)$ & $20(22,2)$ & \\
\hline 2500 a $2999 \mathrm{~g}$ & $21(23,6)$ & $14(15,6)$ & \\
\hline 3000 a $3499 \mathrm{~g}$ & $43(48,3)$ & $28(31,1)$ & \\
\hline $3500 \mathrm{~g}$ ou mais & $13(14,6)$ & $28(31,1)$ & \\
\hline Peso ao nascer (g) & $3000(576)^{\dagger}$ & $3081(635)^{\dagger}$ & $0,4^{\S}$ \\
\hline Escore Z peso/idade & $-2,18(-2,33 \text { a }-2,04)^{* *}$ & $-2,11(-2,24 a-1,97)^{* *}$ & $0,5^{\S}$ \\
\hline Escore Z comprimento/idade & $-1,98(-2,19 a-1,76)^{* *}$ & $-2,00(-2,19 a-1,81)^{* *}$ & $0,9^{\S}$ \\
\hline Escore Z peso/comprimento & $-1,00(-1,16 a-0,84)^{* *}$ & $-0,91(-1,08 a-0,73)^{*}$ & $0,4^{\S}$ \\
\hline Peso/idade & & & 0,2 \\
\hline Abaixo do Percentil 3 & $69(62,2)$ & $55(53,9)$ & \\
\hline Entre P3 e P10 & $42(37,8)$ & $47(46,1)$ & \\
\hline Comprimento/idade & & & 0,7 \\
\hline Abaixo do Percentil 3 & $54(48,6)$ & $49(48,0)$ & \\
\hline Entre P3 e P10 & $27(24,3)$ & $29(28,4)$ & \\
\hline Acima do Percentil 10 & $30(27,0)$ & $24(23,5)$ & \\
\hline Consumo atual de leite materno & $24(21,6)$ & $18(17,6)$ & 0,5 \\
\hline
\end{tabular}


nado quase-experimento ${ }^{8}$. Esse tipo de delineamento é considerado um experimento natural em que o processo de alocação para os grupos intervenção e controle ocorre de forma aleatória não deliberada, uma vez que a randomização não é possível. Dessa forma, esse delineamento apresenta as vantagens de um estudo experimental com simplificação logística e menor custo. Deve-se salientar que esse é um estudo de efetividade, pois quando se faz uma alocação experimental prospectiva é impossível não afetar, em algum grau, a implementação do programa. Além disso, as questões éticas presentes em estudos de intervenção foram particularmente importantes nesse projeto onde não seria aceitável a não inclusão deliberada de algumas crianças no programa de suplementação alimentar. Cabe ressaltar que a comparação de base das características biológicas e socioecômicas das crianças incluídas nos grupos intervenção e controle permite a análise controlada de possíveis fatores de confusão que poderiam ter influência na interpretação dos resultados sobre impacto no crescimento infantil.

Ainda, como uma vantagem, deve ser ressaltada a possibilidade de avaliação do estado nutricional das crianças incluídas nos grupos intervenção e controle através de medidas antropométricas estabelecidas com adequada precisão na avaliação de crianças pequenas ${ }^{9}$, bem como através da utilização de métodos isotópicos considerados acurados, práticos, de baixo custo, e que podem ser realizados em condições de campo sem representar risco ou desconforto à criança que está sendo avaliada. Nesse estudo utilizou-se a administração de uma dose de deutério, isótopo não radioativo que é medido em amostra de saliva e permite a avaliação da composição corporal como uma função da quantidade de água total do organismo da criança ${ }^{10}$.

Por outro lado, o delineamento não foi capaz de prever algumas dificuldades inerentes ao estudo. Porém, para cada uma dessas dificuldades buscou-se a melhor solução para a viabilização do projeto.

A primeira dificuldade encontrada foi que na maioria dos municípios não havia o registro das crianças em risco nutricional nos serviços de saúde e, quando havia, esses não eram adequadamente preenchidos. Sendo assim, os trabalhadores de campo foram orientados para a realização de rastreamento de crianças em risco nutricional, acompanhados dos agentes de saúde do Programa de Saúde da Família de cada município. Embora todos os municípios tenham sido contactados previamente, os trabalhadores de campo encontraram dificuldade na localização de crianças que atendessem aos critérios de inclusão. Mesmo com equipes de saúde locais bastante receptivas, em muitos casos a seleção prévia de crianças nos municípios foi inadequada. Considerando esse aspecto, um membro da equipe de pesquisa foi deslocado um ou dois dias antes dos demais entrevistadores ao município, com a finalidade de realizar a identificação de crianças que atendiam aos critérios de inclusão do estudo.

Em relação à logística do estudo, devem ser considerados dois aspectos metodológicos importantes. O primeiro refere-se ao fato de que, sob o ponto de vista epidemiológico, esse estudo é complexo na medida em que são necessárias duas amostras de saliva 2 a 3 horas após a administração de deutério. Sendo assim, cada entrevistador não pode realizar mais do que três entrevistas por dia. Além disso, deve-se considerar que no estado de Alagoas, mesmo com pequena extensão territorial, as distâncias e dificuldades de acesso aos municípios selecionados tornaram oneroso o custo em transporte e tempo despendido em locomoção.

Finalmente, outro aspecto que deve ser salientado refere-se à não identificação do número de crianças por município que atendessem aos critérios de inclusão. Se por um lado isso pode ser considerado como uma dificuldade do estudo, por outro está de acordo com a redução na ocorrência de déficits nutricionais em crianças brasileiras ${ }^{11}$.

Concluindo esse artigo deve-se enfatizar que, de certa forma, garantiu-se a comparabilidade entre municípios dos grupos inter- 
venção ou controle, uma vez que as crianças que foram incluídas no estudo de base são comparáveis quanto à distribuição demográfica e as características nutricionais. No que se refere à situação socioeconômica, os resultados preliminares mostram que, naturalmente, as equipes municipais estão atendendo, entre os desnutridos, aqueles pertencentes às famílias mais miseráveis. $\mathrm{E}$, finalmente, espera-se que na segunda fase do estudo, quando será realizada análise ajus- tada para esses fatores que se mostraram diferentes entre os grupos no estudo de base, seja possível descortinar o real efeito de um programa de suplementação alimentar sobre o crescimento de crianças desnutridas.

\section{Agradecimentos}

Às Secretarias Municipais e Estadual de Alagoas por sua colaboração durante a realização deste estudo

\section{Referências}

1. INAN/FIBGE/IPES. Pesquisa Nacional Sobre Saúde e Nutrição: Resultados preliminares. Brasília (DF): INAN; 1990.

2. BEMFAM/IBGE/MS/DHS/FNUAP/UNICEF. Pesquisa Nacional Sobre Demografia e Saúde, 1996. Relatório Preliminar. Rio de Janeiro: BEMFAM; 1997.

3. National Center for Health Statistics. Growth Curves for Children, Birth-18 years. United States. Department of Health, Education and Welfare (PHS) Publ. 78-1650 [series 11; 165]. Hyattsville, MD, 1977.

4. Habicht JP, Victora CG, Vaughan JP. Evaluation designs for adequacy, plausibility and probability of public health programme performance and impact. Int J Epidemiol 1999; 28: 10-18.

5. Victora CG, Barros FC, Tomasi E, et al. Tendências e diferenciais na saúde materno-infantil: delineamento e metodologia das coortes de 1982 e 1993 de mães e crianças de Pelotas, Rio Grande do Sul. Cad Saúde Pública 1996; 12 Suppl 1: 7-14.

6. Lohman TG, Roche AF, Martorell R. Anthropometric standardization reference manual. Champaign, Illinois: Human Kinetics Books; 1988.
7. Garrow JS. Índices of adiposity. Nutr Abst Rev Clin Nutr 1983; 53(8): 697-708.

8. Kleinbaum DG, Kupper LL, Morgenstern H. Epidemiologic research: principles and quantitative methods. New York: Lifetime Learning Publications, Van Nostrand Reinhold Company;1982.

9. World Health Organization. Physical status: use and interpretation of anthropometry. Geneva; 1995. (WHO -, Series Technical Report, 854).

10. IAEA. Targeting malnutrition: isotopic tools for evaluating nutrition worldwide. Division of Public Information, Vienna, International Atomic Energy Agency; 1996.

11. Monteiro CA, Benício MHD, Iunes RF, Cardoso MAA. Evolução da desnutrição infantil. In: Monteiro CA. Velhos e novos males de saúde no Brasil. São Paulo: Editora Hucitec - NUPENS/USP; 1995.

Recebido em 04/01/02; aprovado em 15/08/02 